\title{
Gravitational Wave Bursts as Harbingers of Cosmic Strings Diluted by Inflation
}

\author{
Yanou Cui, ${ }^{1, *}$ Marek Lewicki $\oplus^{2,3, \dagger}$ and David E. Morrissey, ${ }^{4, \dagger}$ \\ ${ }^{1}$ Department of Physics and Astronomy, University of California, Riverside, California 92521, USA \\ ${ }^{2}$ Kings College London, Strand, London WC2R 2LS, United Kingdom \\ ${ }^{3}$ Faculty of Physics, University of Warsaw ul. Pasteura 5, 02-093 Warsaw, Poland \\ ${ }^{4}$ TRIUMF, 4004 Wesbrook Mall, Vancouver, British Columbia, Canada V6T 2A3
}

(Received 30 January 2020; revised 30 September 2020; accepted 5 October 2020; published 20 November 2020)

\begin{abstract}
A standard expectation of primordial cosmological inflation is that it dilutes all relics created before its onset to unobservable levels. We present a counterexample to this expectation by demonstrating that a network of cosmic strings diluted by inflation can regrow to a level that is potentially observable today in gravitational waves (GWs). In contrast to undiluted cosmic strings, whose primary GW signals are typically in the form of a stochastic GW background, the leading signal from a diluted cosmic string network can be distinctive bursts of GWs within the sensitivity reach of current and future GW observatories.
\end{abstract}

DOI: 10.1103/PhysRevLett.125.211302

Introduction.-Cosmological observations provide strong support for initial conditions of the form expected from inflation followed by reheating: a hot thermal plasma that is very uniform with small, approximately adiabatic and scale-invariant density fluctuations over distances that are much larger than the Hubble length in that era $[1,2]$. In addition to these features, inflation is expected to have diluted any relics created prior to its beginning, such as superheavy massive particles or most types of topological defects, to negligible levels [3-5]. In this work we investigate cosmic strings as a counterexample to these expectations, and we study the distinctive gravitational wave (GW) signals they produce in this scenario. An observation of such GW signals could provide new information about inflation or potential alternatives.

Cosmic strings are effectively one-dimensional objects that arise in many theories of fundamental physics. They can be fundamental objects [6-10] or configurations of quantum fields such as those originated from a U(1) symmetry breaking $[11,12]$, but at macroscopic distances they are characterized almost completely by their energy per unit length (tension) $\mu$ [13]. In the early universe, cosmic strings are expected to form a network consisting of stable horizon-length long strings together with smaller closed loops that can decay away. The interplay between the slow stretching of long strings and the formation of new loops through string intersections allows a cosmic string

Published by the American Physical Society under the terms of the Creative Commons Attribution 4.0 International license. Further distribution of this work must maintain attribution to the author(s) and the published article's title, journal citation, and DOI. Funded by SCOAP. network to reach a scaling regime in which the total energy density of the network tracks the dominant source of cosmological energy with a relative fraction on the order of $G \mu$ [14-16], where $G=1 / 8 \pi M_{\mathrm{Pl}}^{2}$ is Newton's constant and $M_{\mathrm{Pl}}$ is the reduced Planck mass.

For cosmic strings created after inflation but well before today, scaling is predicted to be achieved reasonably soon after the formation of the network. To maintain scaling energy must be transferred from the network to radiation, and for local or fundamental strings this is usually expected to be in the form of gravitational waves emitted by closed string loops [17-23] (although see Refs. [24-26] that argue for mainly particle emission). As a result, the most promising observational signal from such strings can be the stochastic gravitational wave background (SGWB) created by the combined and unresolved emission of GWs by closed string loops over the history of the cosmos [27-35]. The characteristic frequency spectrum produced this way could also be used to probe the expansion history of the Universe [36-40]. In addition to the SGWB, more recent bursts of GWs from cusps or kinks on string loops can potentially be resolved as individual events [41-44], but for standard scaling strings they are harder to find than the unresolved SGWB [30,32,34].

The situation can be much different for a cosmic string network formed before or shortly after the start of inflation. Like any other relic, such strings would be exponentially diluted by the subsequent inflationary expansion. However, following inflation the energy density in long cosmic strings only falls off as $a^{-2}$, where $a(t)$ is cosmological scale factor, which is much slower than the $a^{-4}$ dilution of radiation and $a^{-3}$ of matter that are expected to dominate the energy budget of the cosmos until close to the present. This difference in dilution rates implies that a network of 
strings can potentially regrow and achieve scaling by today. In such a string scenario, Ref. [45] showed that the SGWB is very strongly suppressed at moderate to high frequencies relative to a string network created after inflation, making it more difficult to observe. For very strong inflationary dilution, the SGWB can also be suppressed at the lower frequencies probed by pulsar timing arrays such as the Parkes pulsar timing array (PPTA) [46] allowing for larger string tensions $G \mu$ to be consistent with existing bounds [47-49].

In this Letter we study the regrowth of cosmic strings after inflationary dilution and we investigate ways to discover them. We demonstrate that individual resolved bursts of GWs can be the leading signal of such strings, and that such bursts could potentially be observed in the current LIGO experiment [50-52], or planned future experiments such as LISA [53], ET [54,55], AION/MAGIS [56-58], and AEDGE [59]. This is also true in cases where the SGWB is significantly suppressed at frequencies relevant to pulsar timing and thus reducing the limits from PPTA [46] and the future reach of the planned Square Kilometer Array (SKA) [60].

Inflation and string regrowth.-To estimate the dilution of cosmic strings formed in the early stages of inflation and their subsequent evolution, we use a simplified picture of inflation and reheating together with the velocity-dependent one-scale (VOS) model to describe the long-string network [61-65].

During inflation we assume a constant Hubble parameter $H_{I}=V_{I} / 3 M_{\mathrm{Pl}}^{2}$ with $V_{I} \equiv M^{4}$ describing the inflationary energy density from initial time $t_{I}$ to end time $t_{E}$. Current observations limit $M \lesssim 10^{16} \mathrm{GeV}$ [2] and we note that $G M^{2} \simeq 7 \times 10^{-7}$. The cosmological scale factor grows as $a(t) \propto e^{H_{I} t}$. After inflation, we assume a reheating period that initiates a radiation-dominated phase with temperature $T_{R H} \leq\left(30 / \pi^{2} g_{*}\right)^{1 / 4} M$. We assume further that the temperature during reheating remains low enough to avoid the destruction of the preexisting cosmic strings by thermal processes such as symmetry restoration.

In the VOS model that we use to describe horizon-length long strings during and after inflation [45], the long string energy is characterized by a correlation length parameter $L$ and velocity parameter $\bar{v}$ such that the energy density of long strings is given by [13]

$$
\rho_{\infty} \equiv \frac{\mu}{L^{2}} .
$$

These parameters evolve according to $[62,63]$

$$
\begin{gathered}
\frac{d L}{d t}=\left(1+\bar{v}^{2}\right) H L+\frac{\tilde{c} \bar{v}}{2}, \\
\frac{d \bar{v}}{d t}=\left(1-\bar{v}^{2}\right)\left[\frac{k(\bar{v})}{L}-2 H \bar{v}\right],
\end{gathered}
$$

where

$$
k(\bar{v})=\frac{2 \sqrt{2}}{\pi}\left(1-\bar{v}^{2}\right)\left(1+2 \sqrt{2} \bar{v}^{3}\right)\left(\frac{1-8 \bar{v}^{6}}{1+8 \bar{v}^{6}}\right),
$$

and $\tilde{c} \simeq 0.23$ describes closed loop formation [63].

As initial condition, we take

$$
L\left(t_{F}\right) \equiv L_{F}=\frac{1}{\zeta H_{I}},
$$

where $t_{F}$ is the greater of the beginning of inflation or the network formation time, and $\zeta^{2}$ corresponds approximately to the number of long strings within the Hubble volume at time $t_{F}$. After $t_{F}$, the string network parameters quickly reach an attractor solution during inflation (independent of the value of $\bar{v}$ at $t_{F}$ ) given by

$$
L(t)=L_{F} e^{H_{I}\left(t-t_{F}\right)}, \quad \bar{v}(t)=\frac{2 \sqrt{2}}{\pi} \frac{1}{H_{I} L(t)} .
$$

This solution reflects the dilution of the long string network, with $H L \gg 1$ and $\bar{v} \ll 1$ by the end of inflation. The subsequent evolution of the string network after inflation takes a very simple form while $H L \gg 1$ with $(L / a)$ approximately constant. It follows that $H L$ decreases after inflation, corresponding to the gradual regrowth of the string network. If the network is to produce a potentially observable signal in GWs, at least a few strings are needed within our current Hubble volume corresponding to $H L \lesssim 1$ today.

To determine the conditions under which there is enough string regrowth for $H L \rightarrow 1$ while also maintaining a sufficient amount of inflation, it is useful to compare the evolution of $L$ prior to scaling to that of the curvature radius $R=1 /(H \sqrt{|\Omega-1|})$ which evolves in precisely the same way, independently of the details of inflation or reheating. In the absence of strong tuning of the curvature radius at the start of inflation, the current $95 \%$ limit on curvature $\left|\Omega_{0}-1\right|=$ $0.0007 \pm 0.0037$ [1] puts the strongest lower bound on the total number of $e$-foldings of inflationary expansion [2]. Defining $\Delta N \geq 0$ to be the number of $e$-foldings between $t_{I}$ and $t_{F}$, the total number of inflationary $e$-foldings is $N_{\text {tot }}=H_{I}\left(t_{E}-t_{I}\right) \equiv N_{F}+\Delta N$. Note that $\Delta N=0$ corresponds to the string forming before or at the start of inflation. Applying the curvature limit on $N_{\text {tot }}$, we find

$$
\begin{aligned}
& \Delta N+\ln \zeta \geq 2.7+\frac{1}{2} \ln \left(\left|\Omega_{I}-1\right|\right) \\
& \quad+\frac{1}{2} \ln \left[\Omega_{\Lambda}(1+\tilde{z})^{-2}+\Omega_{m}(1+\tilde{z})+\Omega_{r}(1+\tilde{z})^{2}\right],
\end{aligned}
$$

where $\tilde{z}$ is the redshift at which $H L \rightarrow 1, \Omega_{a}$ are the fractional energy densities in dark energy, matter, and radiation relative to critical today, $\left|\Omega_{I}-1\right|$ is the deviation from flatness at the 


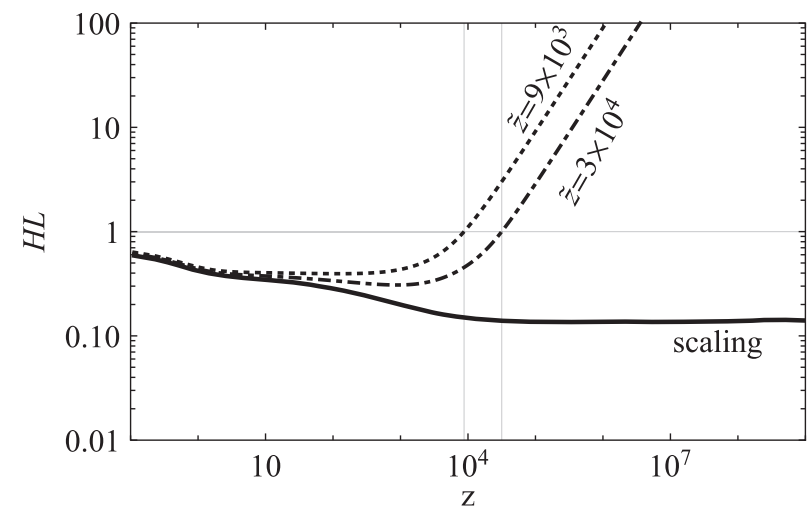

FIG. 1. Evolution of the VOS model string length parameter $H L$ for diluted strings compared to that of a scaling string network for two representative values of the transition redshift $\tilde{z}=9 \times 10^{3}, 3 \times 10^{4}$ at which $H L \rightarrow 1$.

start of inflation, $\ln \left(\left|\Omega_{0}-1\right|\right) / 2 \leq 2.7$ is the bound from Planck [1], and the last line describes the additional evolution of $(\Omega-1)$ between $\tilde{z}$ and now.

To fulfill this bound the network must either have been formed $\Delta N e$-folds after the start of inflation or the initial number of long strings within the Hubble volume at time $t_{I}$ must have been much greater than unity. One or both of these features can arise if the string network was formed in a (weakly) first-order phase transition. Such a transition can produce an initial correlation length much shorter than the Hubble radius, potentially yielding many strings within the Hubble volume at formation and thus $\zeta \gg 1$ [66]. Furthermore, a strong supercooled transition can be initially slow relative to $t_{I} \sim 1 / H_{I}$, in which case it will not complete until after several $e$-folds of expansion to yield $\Delta N>0$ [67-70]. We defer a detailed study of the realization of such conditions within specific theories of inflation and string formation to a future work, although we note that the supercooled-transition scenario of Ref. [68] finds values as large as $\Delta N \sim 18$ as well as the previous studies of string creation in the early stages of inflation of Refs. [47,48,71-75].

String scaling and loop formation.-Once $H L$ approaches unity at redshift $\tilde{z}$, the VOS string length parameter $L$ begins to deviate from the simple $L \propto a$ form and evolves together with $\bar{v}$ toward the string scaling limit. In general, we find that the network does not reach scaling until considerably later than $\tilde{z}$. This is shown in Fig. 1 for two representative values of $\tilde{z}=9 \times 10^{3}, 3 \times 10^{4}$. Using Eq. (7), we find that these values of $\tilde{z}$ correspond to $\Delta N+$ $\ln \zeta \geq 7.3$ and 8.4 which are plausible in the context of supercooled scenarios such as that of Ref. [68].

To determine the density distribution of closed string loops, we use the VOS model described above to find the total rate of loop production prior to and during the scaling regime together with the results of simulations to estimate their initial size. Recent simulations find that (NambuGoto) string scaling networks produce a population of larger loops that are moderately nonrelativistic with initial size $l_{i}=\alpha L\left(t_{i}\right)$ with $\alpha \sim 0.1$ as well as a collection of smaller loops that are highly relativistic [28]. Most of the energy transferred to the smaller loops is in the form of kinetic energy that simply redshifts away, and thus larger loops are expected to be the dominant source of GWs and we focus exclusively on them. The large loops are found to make up a fraction $\mathcal{F}_{\alpha} \sim 0.1$ of the total energy transferred to loops $[28,30]$. After formation, these large loops oscillate, emit energy in the form of GWs, and gradually shorten according to

$$
l(t)=\alpha L\left(t_{i}\right)-\Gamma G \mu\left(t-t_{i}\right),
$$

where $\Gamma \simeq 50$ is the total rate of GW emission [13,20,30]. Matching these simulation results to the total rate of energy loss to loop formation within the VOS model, corresponding to the $\tilde{c} \bar{v} / 2$ term in Eq. (2), the differential number density of long loops per unit length is $[34,65]$

$$
n(l, t) \equiv \frac{\mathcal{F}_{\alpha}}{\sqrt{2}} \frac{[z(t)+1]^{3} /\left[z\left(t_{i}\right)+1\right]^{3}}{\alpha d L /\left.d t\right|_{t=t_{i}}+\Gamma G \mu} \frac{\tilde{c} \bar{v}\left(t_{i}\right)}{\alpha L^{4}\left(t_{i}\right)},
$$

where $t_{i}$ on the right-hand side is to be determined in terms of $l$ and $t$ through Eq. (8).

Gravitational wave signals from string loops.-String loops oscillate and lose energy to GWs. Much of the emission to GWs comes from short, violent, collimated bursts involving cusps or kinks on the string loops. Bursts emitted by a cosmic string network over its cosmic history that are not resolved contribute to the characteristic stochastic gravitational wave background of the network. More recent bursts can also potentially be observed as distinct, individual events. In this section we calculate the GW burst signal following the methods of Refs. [41-43] together with the refinements of Refs. [32,34,44].

The GWs produced by a burst from a cusp or a kink are mostly collimated within a beaming angle,

$$
\theta_{m}(l, z, f)=[(1+z) f l]^{-1 / 3}<1,
$$

where $f$ is the GW frequency seen today and $z$ is the redshift at emission. Within this angular region, the GW waveform is [41-43]

$h(l, z, f)=\frac{f^{-q} l^{2-q}}{(1+z)^{q-1}} \frac{G \mu}{r(z)}, \quad r(z)=\int_{0}^{z} \frac{d z^{\prime}}{H\left(z^{\prime}\right)}$,

where $r(z)$ is the proper distance to the source and $q=4 / 3$ $(5 / 3)$ for cusps (kinks).

The rate of cusp or kink features per unit volume per unit string length at emission is [44]

$$
\nu(l, z)=\frac{2}{l} N_{q} n(l, z),
$$


where $N_{q}$ is the number of features within an oscillation period and $n(l, z)$ is the loop density obtained above. Putting these pieces together, the rate of bursts per volume per length observed today is [32]

$$
\begin{aligned}
& \frac{d^{2} R}{d V d l}(l, a, f) \\
& \quad=\frac{\nu(l, z)}{(1+z)}\left[\frac{\theta_{m}(l, z, f)}{2}\right]^{3(2-q)} \Theta\left(1-\theta_{m}\right) .
\end{aligned}
$$

It is convenient to use

$$
d V=\frac{4 \pi r^{2}(z)}{(1+z)^{3} H(z)} d z
$$

and Eq. (11) to rewrite Eq. (13) as

$$
\frac{d^{2} R}{d z d h}=\frac{2^{3(q-1)} \pi G \mu N_{q}}{(2-q)} \frac{r(z)}{(1+z)^{5} H(z)} \frac{n(l, z)}{h^{2} f^{2}},
$$

where $l$ is now a function of $h, f$, and $z$, and the consistency constraints $\theta_{m}<1$ and $l<\alpha L(z)$ are enforced by restricting $h \in\left[h_{\min }, h_{\max }\right]$ with

$h_{\min }=\frac{1}{(1+z) f^{2}} \frac{G \mu}{r(z)}, \quad h_{\max }=\frac{[\alpha L(z)]^{q-2}}{f^{q}(1+z)^{q-1}} \frac{G \mu}{r(z)}$.

Since the total GW signal is expected to be dominated by bursts from cusps [30,31], we set $q=4 / 3$ and $N_{q}=2.13$ (to match $\Gamma=50$ [34]) in the analysis to follow.

To compare these GW signals with current and future detectors, we separate the contributions into more recent bursts of large amplitude that can be resolved individually from earlier ones that are not resolved and contribute to the net stochastic background. If a burst is to be resolved in a given frequency band $f$, it must produce a strain greater than the experimental sensitivity $h>h_{\exp }$ with rate less than $f$. The rate of such events is $[34,44]$

$$
R_{\exp }(f)=\int_{0}^{z_{*}} d z \int_{\max \left(h_{\min }, h_{\text {exp }}\right)}^{h_{\max }} d h \frac{d^{2} R}{d z d h}(h, z, f),
$$

where $z_{*}$ enforces the rate condition and is given by

$$
f=\int_{0}^{z_{*}} d z \int_{h_{\min }}^{h_{\max }} d h \frac{d^{2} R}{d z d h}(h, z, f) .
$$

Unresolved bursts contribute to the SGWB as [34,44]

$\Omega_{\mathrm{GW}}(f)=\frac{4 \pi^{2} f^{3}}{3 H_{0}^{2}} \int_{z_{*}}^{\infty} d z \int_{h_{\min }}^{h_{\max }} d h h^{2} \frac{d^{2} R}{d z d h}(h, z, f)$,

with $\Omega_{\mathrm{GW}}=\left(f / \rho_{c}\right) d \rho_{\mathrm{GW}} / d f$ for critical density $\rho_{c}$.

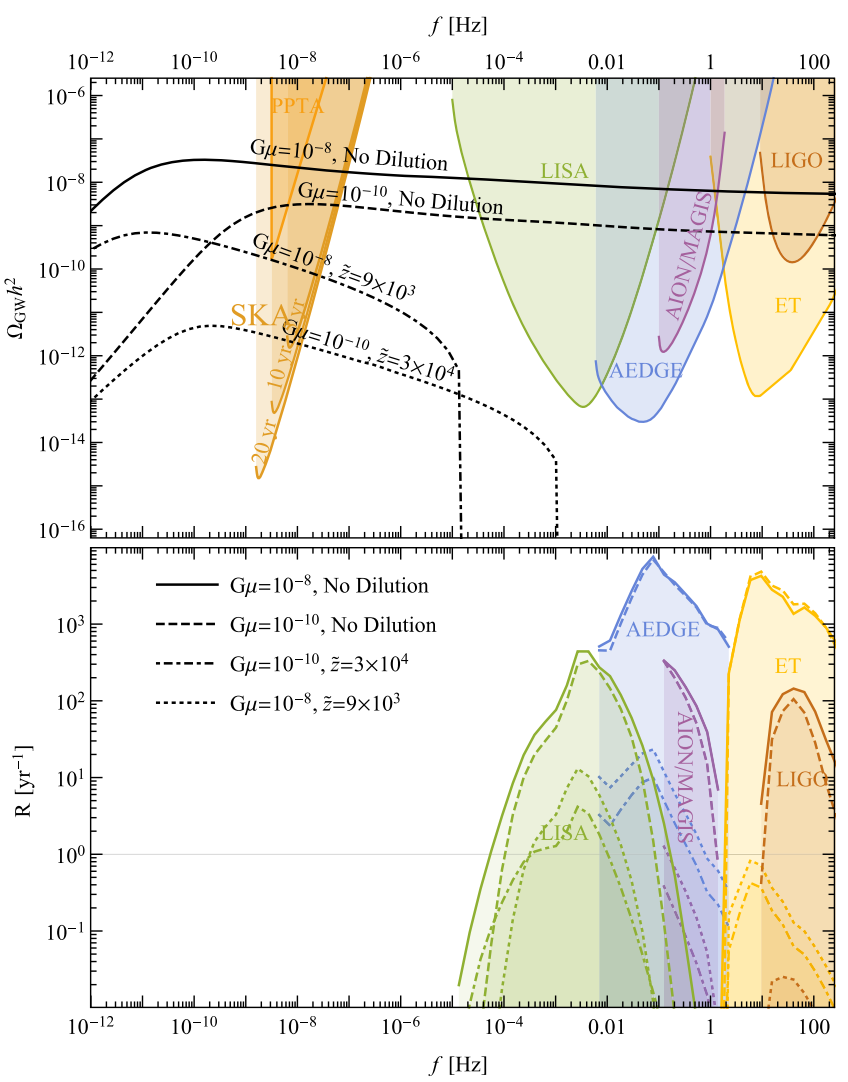

FIG. 2. Gravitational wave signals from diluted and undiluted cosmic string networks as a function of frequency observed today together with the corresponding sensitivities of the indicated observatories. The top panel shows the stochastic GW background while the lower panel gives the event rates of resolved bursts. In both panels we show curves for $G \mu=10^{-8}, 10^{-10}$ for undiluted networks as well as for two diluted networks with $\tilde{z}=9 \times 10^{3}$ for $G \mu=10^{-8}$, and $\tilde{z}=3 \times 10^{-4}$ for $G \mu=10^{-10}$.

In Fig. 2 we show the SGWB from unresolved bursts (top panel) as well as the resolved burst rate as a function of frequency (bottom panel) for diluted and undiluted cosmic string networks. Also shown in these figures are the expected sensitivity ranges of various GW observatories including the current LIGO [52] and planned LISA [53], ET [54,55], AION/MAGIS [56-58], and AEDGE [59], as well as the existing PPTA [46] and planned SKA [60] pulsar timing arrays. The top panel of Fig. 2 shows that undiluted string networks with $G \mu=10^{-8}, 10^{-10}$ are already excluded by pulsar timing measurements at the PPTA [46], but they can be consistent with diluted strings [47]. Despite this strong suppression of the SGWB, the lower panel of Fig. 2 shows that resolved burst events from diluted string networks could still be seen in future gravitational wave observatories.

We note that the SGWB for the diluted networks computed using the burst method described above falls off as $f^{-1 / 3}$ at high frequency, in contrast to the $f^{-1}$ behavior found in Refs. [40,45] computed in a different 


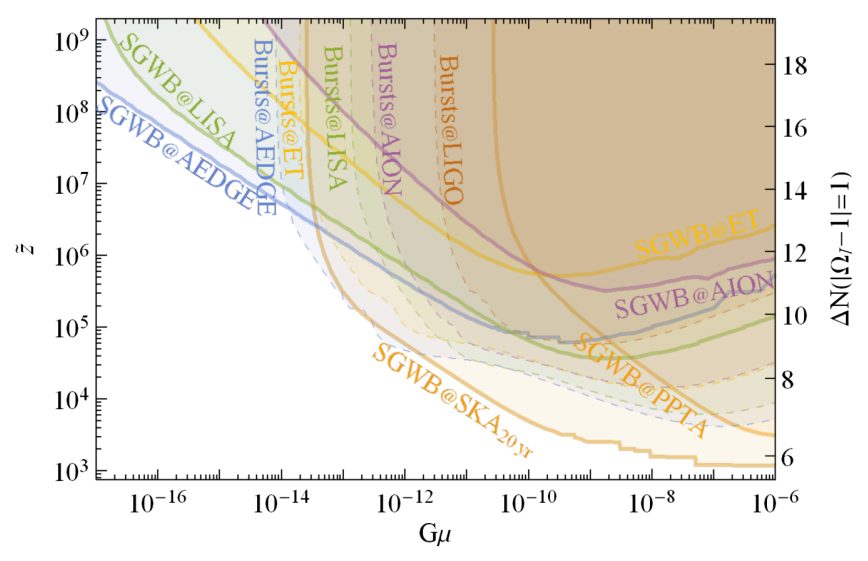

FIG. 3. Sensitivity of current and future GW experiments to signals from diluted cosmic strings as a function of tension $G \mu$ and the redshift $\tilde{z}$ at which loop production resumes. Solid lines show the sensitivity of the indicated experiments to stochastic backgrounds (with $\mathrm{SNR} \geq 10$ ) while dashed lines show their sensitivity to burst signals (with rate $R \geq 1 / \mathrm{yr}$ ).

way by summing over averaged loop normal mode emissions. These two methods predict very similar SGWB signals from cosmic strings in the scaling regime [32,34]. This discrepancy appears to be the result of not including a sufficient number of modes when computing the SGWB with the normal mode method. In the Supplemental Material [76], we show that the number of normal modes needed to compute the SGWB for a diluted cosmic string network can be orders of magnitude larger than for a cosmic string that reaches scaling very early, and that when a large number is required the signal computed with the normal mode method asymptotes to $\Omega_{G W} \propto f^{-1 / 3}$, in agreement with the burst method used in this work. At higher frequencies, we also find a sharper drop from the burst method caused by the subtraction of infrequent bursts from the SGWB.

To illustrate the potential observability of diluted strings for more general scenarios, we show in Fig. 3 the reach of current and future GWs with related detectors as a function of the string tension $G \mu$ and redshift $\tilde{z}$ when strings grow back into the horizon. The figure demonstrates that bursts can be the leading discovery channel for a broad range of string tensions and dilution factors, with the SGWB signal becoming more prominent for larger $\tilde{z}$ corresponding to less dilution. For very strong dilution with $\tilde{z} \lesssim 10^{3}$, the string network may not have reached scaling even by the present time and the combined GW signal is too weak to be observed in the foreseeable future. With such a dilution, other direct bounds such as the CMB distortion limit of $G \mu<1.1 \times 10^{-7}$ [81] are expected to be mitigated as well, although such a low $\tilde{z}$ regime may still be observable in late-time astrophysical effects such as gravitational lensing and imprints on structure formation [49,82-85].

Conclusions. - In this Letter we have demonstrated that a network of cosmic strings formed before or in the early stages of primordial inflation can regrow to observable levels today. Furthermore, we have shown that in this scenario the stochastic gravitational wave signal is strongly suppressed and the most promising discovery channel can be distinctive burst signals in gravitational wave detectors.

We thank Jose Juan Blanco-Pillado, Djuna Croon, James Wells, and Graham White for helpful discussions. Y. C. is supported in part by the U.S. Department of Energy under Award No. DE-SC0008541. M. L. is supported by the UK STFC Grant No. ST/P000258/1 and the Polish National Science Center Grant No. 2018/31/D/ST2/02048. D. E. M. is supported by a Discovery Grant from the Natural Sciences and Engineering Research Council of Canada (NSERC), and TRIUMF, which receives federal funding via a contribution agreement with the National Research Council of Canada (NRC).

Note added.-Our results recently also gained experimental relevance as the NANOGrav Collaboration reported strong evidence for a stochastic common-spectrum process [86], which can be interpreted as SGWB from cosmic strings $[87,88]$. In fact, our current results have a slight advantage over the vanilla cosmic string case as the current data favor a negative slope for the GW abundance which we generically obtain in our scenario at pulsar timing array frequencies. We have in fact verified that our first benchmark point $G \mu=10^{-8}, \tilde{z}=9 \times 10^{3}$ fits this new data at 95\% C.L. and a stronger signal would improve the fit.

*yanou.cui@ucr.edu

marek.lewicki@kcl.ac.uk

tmorri@triumf.ca

[1] N. Aghanim et al. (Planck Collaboration), Astron. Astrophys. 641, A6 (2020).

[2] Y. Akrami et al. (Planck Collaboration), Astron. Astrophys. 641, A10 (2020).

[3] A. H. Guth, Phys. Rev. D 23, 347 (1981).

[4] A. D. Linde, Phys. Lett. 108B, 389 (1982).

[5] A. Albrecht and P. J. Steinhardt, Phys. Rev. Lett. 48, 1220 (1982).

[6] E. J. Copeland, R. C. Myers, and J. Polchinski, J. High Energy Phys. 06 (2004) 013.

[7] G. Dvali and A. Vilenkin, J. Cosmol. Astropart. Phys. 03 (2004) 010.

[8] J. Polchinski, in Proceedings of the NATO Advanced Study Institute on String Theory: From Gauge Interactions to Cosmology, Cargese, France, 2004 (Springer, Doredrecht, The Netherlands, 2004), pp. 229-253.

[9] M. G. Jackson, N. T. Jones, and J. Polchinski, J. High Energy Phys. 10 (2005) 013.

[10] S. H. H. Tye, I. Wasserman, and M. Wyman, Phys. Rev. D 71, 103508 (2005); 71, 129906(E) (2005).

[11] H. B. Nielsen and P. Olesen, Nucl. Phys. B61, 45 (1973).

[12] T. W. B. Kibble, J. Phys. A 9, 1387 (1976). 
[13] A. Vilenkin and E. P. S. Shellard, Cosmic Strings and Other Topological Defects (Cambridge University Press, Cambridge, England, 2000).

[14] A. Albrecht and N. Turok, Phys. Rev. Lett. 54, 1868 (1985).

[15] D. P. Bennett and F. R. Bouchet, Phys. Rev. Lett. 60, 257 (1988).

[16] B. Allen and E. P. S. Shellard, Phys. Rev. Lett. 64, 119 (1990).

[17] A. Vilenkin, Phys. Lett. 107B, 47 (1981).

[18] T. Vachaspati and A. Vilenkin, Phys. Rev. D 31, 3052 (1985).

[19] N. Turok, Nucl. Phys. B242, 520 (1984).

[20] C. J. Burden, Phys. Lett. 164B, 277 (1985).

[21] K. D. Olum and J. J. Blanco-Pillado, Phys. Rev. Lett. 84, 4288 (2000).

[22] J. N. Moore, E. P. S. Shellard, and C. J. A. P. Martins, Phys. Rev. D 65, 023503 (2001).

[23] D. Matsunami, L. Pogosian, A. Saurabh, and T. Vachaspati, Phys. Rev. Lett. 122, 201301 (2019).

[24] G. Vincent, N. D. Antunes, and M. Hindmarsh, Phys. Rev. Lett. 80, 2277 (1998).

[25] N. Bevis, M. Hindmarsh, M. Kunz, and J. Urrestilla, Phys. Rev. D 75, 065015 (2007).

[26] D. G. Figueroa, M. Hindmarsh, and J. Urrestilla, Phys. Rev. Lett. 110, 101302 (2013).

[27] B. Allen, in Relativistic Gravitation and Gravitational Radiation, Proceedings of the Les Houches School of Physics, Les Houches, France, 1995 (Cambridge University Press, Cambridge, England, 1996), pp. 373-417.

[28] J. J. Blanco-Pillado, K. D. Olum, and B. Shlaer, Phys. Rev. D 89, 023512 (2014).

[29] J. J. Blanco-Pillado, K. D. Olum, and X. Siemens, Phys. Lett. B 778, 392 (2018).

[30] J. J. Blanco-Pillado and K. D. Olum, Phys. Rev. D 96, 104046 (2017).

[31] C. Ringeval and T. Suyama, J. Cosmol. Astropart. Phys. 12 (2017) 027.

[32] B. P. Abbott et al. (LIGO Scientific and Virgo Collaborations), Phys. Rev. D 97, 102002 (2018).

[33] C. Caprini and D. G. Figueroa, Classical Quantum Gravity 35, 163001 (2018).

[34] P. Auclair et al., J. Cosmol. Astropart. Phys. 04 (2020) 034.

[35] P. Auclair, D. A. Steer, and T. Vachaspati, Phys. Rev. D 101, 083511 (2020).

[36] Y. Cui, M. Lewicki, D. E. Morrissey, and J. D. Wells, Phys. Rev. D 97, 123505 (2018).

[37] Y. Cui, M. Lewicki, D. E. Morrissey, and J. D. Wells, J. High Energy Phys. 01 (2019) 081.

[38] C.-F. Chang and Y. Cui, Phys. Dark Universe 29, 100604 (2020).

[39] Y. Gouttenoire, G. Servant, and P. Simakachorn, J. Cosmol. Astropart. Phys. 07 (2020) 016.

[40] Y. Gouttenoire, G. Servant, and P. Simakachorn, J. Cosmol. Astropart. Phys. 07 (2020) 032.

[41] T. Damour and A. Vilenkin, Phys. Rev. Lett. 85, 3761 (2000).

[42] T. Damour and A. Vilenkin, Phys. Rev. D 64, 064008 (2001).

[43] T. Damour and A. Vilenkin, Phys. Rev. D 71, 063510 (2005).
[44] X. Siemens, J. Creighton, I. Maor, S. R. Majumder, K. Cannon, and J. Read, Phys. Rev. D 73, 105001 (2006).

[45] G. S. F. Guedes, P. P. Avelino, and L. Sousa, Phys. Rev. D 98, 123505 (2018).

[46] R. M. Shannon et al., Science 349, 1522 (2015).

[47] K. Kamada, Y. Miyamoto, and J. Yokoyama, J. Cosmol. Astropart. Phys. 10 (2012) 023.

[48] K. Kamada, Y. Miyamoto, D. Yamauchi, and J. Yokoyama, Phys. Rev. D 90, 083502 (2014).

[49] C. Ringeval, D. Yamauchi, J. Yokoyama, and F. R. Bouchet, J. Cosmol. Astropart. Phys. 02 (2016) 033.

[50] J. Aasi et al. (LIGO Scientific Collaboration), Classical Quantum Gravity 32, 115012 (2015).

[51] E. Thrane and J. D. Romano, Phys. Rev. D 88, 124032 (2013).

[52] B. P. Abbott et al. (Virgo and LIGO Scientific Collaborations), Phys. Rev. Lett. 116, 131102 (2016).

[53] N. Bartolo et al., J. Cosmol. Astropart. Phys. 12 (2016) 026.

[54] M. Punturo et al., Classical Quantum Gravity 27, 194002 (2010).

[55] S. Hild et al., Classical Quantum Gravity 28, 094013 (2011).

[56] P. W. Graham, J. M. Hogan, M. A. Kasevich, and S. Rajendran, Phys. Rev. D 94, 104022 (2016).

[57] P. W. Graham, J. M. Hogan, M. A. Kasevich, S. Rajendran, and R. W. Romani (MAGIS Collaboration), arXiv:1711.02225.

[58] O. Buchmuller, J. Cosmol. Astropart. Phys. 05 (2020) 011.

[59] Y. A. El-Neaj et al. (AEDGE Collaboration), Eur. Phys. J. Quantum Technol. 7, 6 (2020).

[60] G. Janssen et al., Proc. Sci., AASKA14 (2015) 037.

[61] C. J. A. P. Martins and E. P. S. Shellard, Phys. Rev. D 53, R575 (1996).

[62] C. J. A. P. Martins and E. P. S. Shellard, Phys. Rev. D 54, 2535 (1996).

[63] C. J. A. P. Martins and E. P. S. Shellard, Phys. Rev. D 65, 043514 (2002).

[64] P. P. Avelino and L. Sousa, Phys. Rev. D 85, 083525 (2012).

[65] L. Sousa and P. P. Avelino, Phys. Rev. D 88, 023516 (2013).

[66] A. Rajantie, Int. J. Mod. Phys. A 17, 1 (2002).

[67] M. S. Turner, E. J. Weinberg, and L. M. Widrow, Phys. Rev. D 46, 2384 (1992).

[68] T. Konstandin and G. Servant, J. Cosmol. Astropart. Phys. 12 (2011) 009.

[69] J. Ellis, M. Lewicki, and J. M. No, J. Cosmol. Astropart. Phys. 04, (2019) 003.

[70] J. Ellis, M. Lewicki, J. M. No, and V. Vaskonen, J. Cosmol. Astropart. Phys. 06 (2019) 024.

[71] Q. Shafi and A. Vilenkin, Phys. Rev. D 29, 1870 (1984).

[72] E. T. Vishniac, K. A. Olive, and D. Seckel, Nucl. Phys. B289, 717 (1987).

[73] J. Yokoyama, Phys. Rev. Lett. 63, 712 (1989).

[74] H. M. Hodges and J. R. Primack, Phys. Rev. D 43, 3155 (1991).

[75] R. Basu, A. H. Guth, and A. Vilenkin, Phys. Rev. D 44, 340 (1991).

[76] See Supplemental Material at http://link.aps.org/ supplemental/10.1103/PhysRevLett.125.211302 for discussion of resummation of normal modes needed to accurately compute the SGWB, which includes Refs. [77-80].

[77] S. A. Sanidas, R. A. Battye, and B. W. Stappers, Phys. Rev. D 85, 122003 (2012). 
[78] J. J. Blanco-Pillado, K. D. Olum, and B. Shlaer, Phys. Rev. D 92, 063528 (2015).

[79] L. Sousa, P. P. Avelino, and G. S. F. Guedes, Phys. Rev. D 101, 103508 (2020).

[80] S. Blasi, V. Brdar, and K. Schmitz, arXiv:2004.02889.

[81] T. Charnock, A. Avgoustidis, E. J. Copeland, and A. Moss, Phys. Rev. D 93, 123503 (2016).

[82] M. V. Sazhin, O. S. Khovanskaya, M. Capaccioli, G. Longo, M. Paolillo, G. Covone, N. A. Grogin, and E. J. Schreier, Mon. Not. R. Astron. Soc. 376, 1731 (2007).
[83] E. Morganson, P. Marshall, T. Treu, T. Schrabback, and R. D. Blandford, Mon. Not. R. Astron. Soc. 406, 2452 (2010).

[84] S. Laliberte, R. Brandenberger, and D. C. N. da Cunha, arXiv:1807.09820.

[85] M. A. Fernandez, S. Bird, and Y. Cui, Phys. Rev. D 102, 043509 (2020).

[86] Z. Arzoumanian et al. (NANOGrav Collaboration), arXiv:2009.04496.

[87] J. Ellis and M. Lewicki, arXiv:2009.06555.

[88] S. Blasi, V. Brdar, and K. Schmitz, arXiv:2009.06607. 